\title{
Physical Activity and Cardiovascular Health: Practical Strategies to Reduce Sedentary Time in Adult Population
}

\author{
Karolinny Borinelli de Aquino Moura, ${ }^{\circledR}$ Simone Savaris, ${ }^{(0)}$ Janice Debastiani, ${ }^{\circledR}$ Juliana Beust de Lima ${ }^{(\bullet)}$ \\ Universidade Federal do Rio Grande do Sul, Porto Alegre, RS - Brazil
}

\section{Introduction}

Cardiovascular disease (CVD) is highly prevalent worldwide and is the main cause of death in the general population. ${ }^{1}$ The practice of physical activity (PA) has emerged as a primary prevention strategy for CVD, with the potential to control risk factors, and promote a better quality of life and longer survival. ${ }^{2}$

However, despite the scientifically proven benefits, and formal recommendations for the practice of PA for adults, ${ }^{3}$ (Table 1), published data on the world population are alarming. Recent estimates indicate that one in four $(27.5 \%)$ adults worldwide and almost half of the population in Brazil do not follow the recommendations made by the World Health Organization (WHO). ${ }^{4}$ Additionally to physical inactivity, the growing prevalence of sedentary behavior is a current concern ${ }^{5}$ (Table 2).

Prolonged periods of sedentary behavior have been associated with unfavorable health outcomes. On the other hand, the practice of PA according to the WHO recommendations can attenuate or even eliminate the association between sitting time with all-cause and cardiovascular mortality risk. ${ }^{6}$ These findings reinforce the importance of reducing the time of sedentary behavior and mainly practicing higher volumes of PA, particularly in individuals who cannot avoid exposure to prolonged periods of sedentary behaviour. ${ }^{7}$ The current WHO Guidelines on Physical Activity and Sedentary Behavior,

\section{Keywords}

Cardiovascular Diseases/prevention and control; Exercise; Sedentary Behavior; Health Promotion; Activity Physical; Quality of Life; Adult. an update of the previous 2010 recommendations, ${ }^{8}$ in addition to encouraging physical activity practice, strongly recommends reducing the time of sedentary behaviour. ${ }^{3}$ This is reinforced by the recently published Physical Activity Guidelines for the Brazilian population. ${ }^{9}$

In 2018, the WHO developed a global action plan named "More Active People for a Healthier World" aimed at reducing by $15 \%$ the overall prevalence of physical inactivity among adolescents and adults by $2030 .{ }^{10}$ In line with plan, based on the available literature, we suggest simple strategies to reduce the time of sedentary behavior and increase the volume of PA.

\section{Physical activity-based health promotion strategies}

Considering prolonged sedentary behavior can be deleterious to health, the first strategy is to interrupt these periods and to perform some PA. It has been shown that interrupting sitting position during leisure-time more frequently has a positive effect on mental health ${ }^{11}$ and metabolic profile. ${ }^{12}$ Besides that, some experimental studies have shown positive acute responses in vascular health and blood pressure. ${ }^{13,14}$

An accessible and free method to optimize time is using stairs instead of elevators or escalators. Although some deep-rooted habits (such as the use of escalators) can be difficult to replace, stairs are often found in different places and opportunities for climbing stairs are commonly available and do not involve monetary costs. Regarding this strategy, the available evidence points to enhancing cardiorespiratory fitness and improving the lipid profile. ${ }^{15}$ In addition, a cohort study has verified a dose-response association between number of floors climbed and all-cause mortality. ${ }^{16}$

Mailing Address: Juliana Beust de Lima

Rua Ramiro Barcelos, 2350. Postal Code: 90035-007, Porto Alegre, RS - Brazil.

E-mail: julianabeustdelima@gmail.com 
Table 1 - World Health Organization's recommendations

\section{Physical Activity}

1. All adults should undertake regular physical activitya.

2. Adults should do at least 150-300 minutes of moderate-intensity aerobic physical activity; or at least 75-150 minutes of vigorousintensity aerobic physical activity; or an equivalent combination of moderate- and vigorous-intensity activity throughout the week, for substantial health benefits .

3. Adults may increase moderate-intensity aerobic physical activity to more than 300 minutes; or do more than 150 minutes of vigorousintensity aerobic physical activity; or an equivalent combination of moderate- and vigorous-intensity activity throughout the week for additional health benefits ${ }^{\mathrm{b}}$.

4. Adults should also do muscle-strengthening activities at moderate or greater intensity that involve all major muscle groups on two or more days a week, as these provide additional health benefits ${ }^{\text {. }}$

\section{Sedentary Behavior}

1. Adults should limit the amount of time spent being sedentary. Replacing sedentary time with physical activity of any intensity (including light intensity) provides health benefitsa.

2. To help reduce the detrimental effects of high levels of sedentary behavior on health, adults should aim to do more than the recommended levels of moderate- to vigorous-intensity physical activitya.

Strong recommendation, moderate certainty evidence ${ }^{a}$

Conditional recommendation, moderate certainty evidence ${ }^{b}$

Source: Authors. Adapted from WHO Guidelines on Physical Activity and Sedentary Behaviour. Geneva: World Health Organization; 2020.

\section{Table 2 - Key concepts}

\section{Sedentary Behavior}

Any waking behavior characterized by an energy expenditure $\leq 1.5$ metabolic equivalents (METs) while sitting, reclining, or lying ${ }^{3}$.

\section{Physical Activity}

Any bodily movement produced by skeletal muscles that requires energy expenditure ${ }^{3}$.

\section{Physical Inactivity}

An insufficient physical activity level to meet current physical activity recommendations ${ }^{3}$.

Source: Authors. Adapted from WHO Guidelines on Physical Activity and Sedentary Behavior. Geneva: World Health Organization; 2020.

Regarding these potential benefits, another pragmatic strategy is active commuting (for example, walking and cycling), taking the opportunity to practice PA on the pathway between home, school, or work. A prospective study has demonstrated that people who cycled as transportation to work, spending on average three hours per week, had a significant reduction of approximately $30 \%$ in risk of mortality. ${ }^{17}$ Other studies also have shown the association between walking and cycling with reducing the incidence of chronic non-communicable diseases. Thus, this simple, ecologic, and economic strategy could be a relevant measure to be encouraged in concomitance with safety precautions when commuting. ${ }^{18}$
Using technology to promote PA is a smart strategy. There are many useful tools designed to encourage people to exercise: automatic monitoring devices, applications, social media, video games and other software programs. These involve planned, group or individual activities, that may be performed independently of weather conditions, and combine virtual and real environments. ${ }^{19}$

In this scenario, wearables and smartphone applications appear to be a trend in health care. These instruments are effective in promoting PA in adults ${ }^{20}$ and could assist in setting goals, motivating and monitoring different health behaviours. ${ }^{21}$ Considering that these devices enable self-monitoring and data sharing (allowing supervision 
Table 3 - Summarized strategies

\section{Active breaks}

Active interruptions of the sedentary period to perform some PA.

Climbing stairs

Using stairs instead of elevators or escalators.

Active commuting

Active displacement, practicing PA on the way between home and school or work.

Technological tools

Use of technological tools that stimulate PA.

Source: Authors. PA: physical activity

and guidance of health professionals), the combination of these strategies seems to be ideal. ${ }^{20}$ Furthermore, the Köhler effect has been reported in virtual scenarios, i.e., when people compare different performance outcomes with each other, which increases their motivation. ${ }^{22}$

Another interesting technology-based strategy to replace sedentary behavior with movement is using active video games. In addition to increasing PA, evidence points to benefits in several subpopulations and outcomes, including: improvement of physical function and cognition, reduction of depression and body weight. However, evidence suggests that less physically active users are more likely to abandon the use of these devices and return to old sedentary routine over time. Then, the main challenge is to improve adherence of these users ${ }^{19}$ (Table 3).

\section{Conclusion}

For the general population, reducing prolonged periods of sedentary behavior and increasing the volume of PA is primordial. Performing some physical activity, even at lower volumes and intensity than recommended, is better than performing none and will have an impact on health. The measurements and strategies proposed in this article, including active breaks, climbing stairs, active commuting and use of technological tools. are evidence-based behavioral, practical changes to promote the health of the adult population.

\section{Author contributions}

The conception and design of the research was performed by all authors. JBL has written the introduction and about active pauses, SS, JD and JBL about active displacement and KBAM about using technology to promote physical activity. The article translation from Portuguese to English was performed by KBAM and SS. There was no new acquisition of data, analysis and interpretation of new data nor statistical analysis. There was no financial support. Critical revision of the manuscript for intellectual content was performed by all authors. The final version of the manuscript was approved by all authors.

\section{Potential Conflict of Interest}

No potential conflict of interest relevant to this article was reported.

\section{Sources of Funding}

There were no external funding sources for this study.

\section{Study Association}

This study is not associated with any thesis or dissertation work. 


\section{References}

1. GBD 2017 Causes of Death Collaborators. Global, regional, and national age-sex-specific mortality for 282 causes of death in 195 countries and territories, 1980-2017: a systematic analysis for the Global Burden of Disease Study 2017. Lancet. 2018;392(10159):1736-88.

2. Mendis S, Puska P, Norrving B, World Health Organization. Global atlas on cardiovascular disease prevention and control: Geneva:WHO;2011.

3. World Health Organization (WHO). Guidelines on physical activity and sedentary behaviour. Geneva; 2020.

4. Guthold R, Stevens GA, Riley LM, Bull FC. Worldwide trends in insufficient physical activity from 2011 to 2016: a pooled analysis of 358 population-based surveys with 1.9 million participants. Lancet Glob Health. 2018; 6(10):e1077-e1086.

5. Sedentary Behaviour Research Network. Letter to the editor: standardized use of the terms "sedentary" and "sedentary behaviours". Appl Physiol Nutr Metab. 2012;37(3):540-2.

6. Stamatakis E, Gale J, Bauman A, Ekelund U, Hamer M, Ding D. Sitting time, physical activity, and risk of mortality in adults. J Am Coll Cardiol. 2019;73(16):2062-72.

7. Piercy KL, Troiano RP, Ballard RM, Carlson SA, Fulton JE, Galuska DA, et al. The Physical Activity Guidelines for Americans. JAMA. 2018;320(19):2020-8.

8. World Health Organization. (WHO) Global recommendations on physical activity for health. Geneva; 2010.

9. Ministry of Health of Brazil. Secretariat of Primary Health Care. Health Promotion Department. Physical Activity Guidelines for the Brazilian Population. Brazil; 2021.

10. World Health Organization.(WHO) Global action plan on physical activity 2018-2030. More active people for a healthier world. Geneva:2018.

11. Hallgren M, Nguyen TT, Owen N, Vancampion D, Smith L, Dunstan DW, et al. Associations of interruptions to leisure-time sedentary behaviour with symptoms of depression and anxiety. Transl Psychiatry. 2020;10(1):128.

12. Healy GN, Dunstan DW, Salmon J, Cerin E, Shaw JE, Zimmet PZ, et al. Breaks in sedentary time: beneficial associations with metabolic risk. Diabetes Care. 2008;31(4):661-6.
13. Thosar SS, Bielko SL, Mather KJ, Johnston JD, Wallace JP. Effect of prolonged sitting and breaks in sitting time on endothelial function. Med Sci Sports Exerc. 2015;47(4):843-9.

14. Wheeler MJ, Dunstan DW, Ellis KA, Cerin E, Phillips S, Lambert G, et al. Effect of Morning Exercise With or Without Breaks in Prolonged Sitting on Blood Pressure in Older Overweight/Obese Adults. Hypertension. 2019;73(4):859-67.

15. Boreham CA, Kennedy RA, Murphy MH, Tully M, Wallace WF, Young I. Training effects of short bouts of stair climbing on cardiorespiratory fitness, blood lipids, and homocysteine in sedentary young women. $\mathrm{Br}$ J Sports Med. 2005;39(9):590-3.

16. Rey-Lopez JP, Stamatakis E, Mackey M, Sesso HD, Lee IM. Associations of self-reported stair climbing with all-cause and cardiovascular mortality: The Harvard Alumni Health Study. Prev Med Rep. 2019;15:100938.

17. Andersen LB, Schnohr P, Schroll M, Hein HO. All-cause mortality associated with physical activity during leisure time, work, sports, and cycling to work. Arch Intern Med. 2000;160(11):1621-8.

18. Andersen LB. Active commuting is beneficial for health. BMJ Clinical Research.. 2017;357:j1740. Doi:10.1136/bmj1740

19. Tate DF, Lyons EJ, Valle CG. High-Tech Tools for Exercise Motivation: Use and Role of Technologies Such as the Internet, Mobile Applications, Social Media, and Video Games. Diabetes Spectr. 2015; 28(1): 45-54.

20. Hakala S, Rintala A, Immonen J, Karvanen J, Heinonen A, Sjögren T. Effectiveness of physical activity promoting technology-based distance interventions compared to usual care. Systematic review, meta-analysis and meta-regression. Eur J Phys Rehabil Med. 2017; 53(6):953-67.

21. Schoeppe S, Alley S, Van Lippevelde W, Bray NA, Williams SL, Duncan MJ, et al. Efficacy of interventions that use apps to improve diet, physical activity and sedentary behaviour: a systematic review. Int J Behav Nutr Phys Act. 2016;13(1):127.

22. Wattanapisit A, Amaek W, Sukkriang N, Wattanapisit S, Wongsiri S. Perspectives on Using Online Platforms for Promoting Running and Walking Activities. Front Public Health. 2020;8:150. 